\title{
ANALYTICITY OF DETERMINANTS OF OPERATORS ON A BANACH SPACE
}

\author{
JAMES S. HOWLAND ${ }^{1}$
}

\begin{abstract}
If $F(z)$ is an analytic family of operators on a Banach space which is of finite rank for each $z$, then rank $F(z)$ is constant except for isolated points, and det $(I+F(z))$ and $\operatorname{tr} F(z)$ are analytic. Similarly if $F(z)$ is meromorphic.
\end{abstract}

In this note, we consider an analytic family $F(z)$ of operators on an arbitrary complex Banach space $X$, such that the rank of $F(z)$ is finite for each $z$. We prove that the rank of $F(z)$ is constant on the domain of analyticity, except for isolated points, and that the trace of $F(z)$ and the determinant of $I+F(z)$ are analytic functions on that domain. This is not immediately clear, since the range of $F(z)$ need not lie in a fixed finite dimensional subspace which is independent of $z$. The proof uses the trace norm of Ruston [2]. We extend the result to a meromorphic family, and establish the standard formula for the logarithmic derivative of det $(I+F(z))$. For the definitions of trace and determinant, see [2] and [1, pp. 160-162].

1. THEOREM. If $F(z)$ is analytic on a domain $\Omega$ and rank $F(z)$ is finite for each $z$, then there is an integer $m$ such that rank $F(z)=m$ except at isolated points where rank $F(z)<m$.

2. Lemma. If $F \in \mathbb{B}(X)$, then rank $F \geqq N$ iff there exist bounded projections $P$ and $Q$ of dimension $N$ such that $P F Q$ has rank $N$.

PRoof. If rank $F<N$, then rank $P F Q \leqq$ rank $F<N$. Conversely, if rank $F \geqq N$, there are $x_{1}, \cdots, x_{N}$ such that $F x_{1}, \cdots, F x_{N}$ are linearly independent. If $P$ projects on the span of $F x_{1}, \cdots, F x_{N}$ and $Q$ on the span of $x_{1}, \cdots, x_{N}$, then $P F Q$ has rank $N$.

This lemma implies that rank is upper semicontinuous: if $F_{n} \rightarrow F$ in norm and rank $F_{n} \leqq m$, then rank $F \leqq m$. For if $P$ and $Q$ have the same dimension exceeding $m$, then $\operatorname{det} P F Q=\lim \operatorname{det} P F_{n} Q=0$, where the determinants are with respect to fixed bases of $P X$ and $Q X$. (One may also conclude this by considering a determinant of the form $\operatorname{det}\left[\left\langle x_{i}^{*}, F x_{j}\right\rangle\right]$.)

Received by the editors January $24,1970$.

AMS 1969 subject classifications. Primary 4745; Secondary 4748.

Key words and phrases. Trace class of Banach space, operator-valued analytic functions, determinants, finite rank operators.

1 Supported by ARO Grant DA-ARO-D-31-124-G978.

Copyright ( 1971, American Mathematical Society 
Proofof Theorem 1. For each $k \geqq 0$, let $E_{k}=\{z \in \Omega \mid \operatorname{rank} \mathrm{F}(z) \leqq k\}$. Since $\Omega=\cup_{k=1}^{\infty} E_{k}, E_{k}$ is uncountable for some integer $k$, and so there is a smallest integer $m$ such that $E_{m}$ has a point of accumulation within $\Omega$. If $P$ and $Q$ are arbitrary projections with $\operatorname{dim} P=\operatorname{dim} Q>m$, then the determinant $d(z)$ of $P F(z) Q$, computed with respect to fixed bases of $P \mathscr{X}$ and $Q \mathscr{X}$, vanishes on $E_{m}$, and hence on all of $\Omega$. Since $P$ and $Q$ are arbitrary, Lemma 2 implies that $E_{m}=\Omega$. Since $m$ is minimal, $E_{m-1}$ consists of isolated points.

This proof also shows that the rank of $F(z)$ is determined by its values on any set with an accumulation point in $\Omega$, and hence that no analytic continuation of $F(z)$ can have rank exceeding $m$. The hypothesis of Theorem 1 can be weakened by assuming only that the set of points at which $F(z)$ has finite rank is uncountable; however, it does not suffice to assume only that $F(z)$ has finite rank on a set with an accumulation point in $\Omega$, for if $F(z)$ is the infinite diagonal matrix

$$
F(z)=\left(\begin{array}{llll}
a_{1}(z) & & & \\
& a_{2}(z) & & \\
& & \ddots & \\
& & a_{n}(z) & \ddots
\end{array}\right)
$$

where $a_{n}(z)=(z-1)(z-1 / 2) \cdots(z-1 / n)$, then $F(z)$ is analytic for $|z|<1$, while rank $F(1 / n)=n-1$.

If $F \in \mathbb{B}(X)$ has finite rank, then, following Ruston [2], [3] let $\beta(F)$ denote the operator norm of $F$ and

$$
\tau(F)=\inf \sum_{i=1}^{m}\left|x_{i}^{*}\right|\left|x_{i}\right|
$$

where the infimum is taken over all representations $F=\sum_{i=1}^{m}\left\langle x_{i}^{*}, \cdot\right\rangle x_{i}$ of $F . \tau$ is a norm, and

(1) $|\operatorname{tr} F| \leqq \tau(F)$,

(2) $\beta(F) \leqq \tau(F) \leqq \beta(F)$ rank $F$, and

(3) $\tau(A F) \leqq \beta(A) \tau(F)$ for any $A$ in $B(X)$.

3. THEOREM. If $F(z)$ is analytic and the rank of $F(z)$ is finite for all $z$ in $\Omega$, then $\operatorname{tr} F(z)$ is analytic, and $d \operatorname{tr} F(z) / d z=\operatorname{tr} F^{\prime}(z)$.

Proof. By Theorem 1, rank $F(z) \leqq m<\infty$ for some integer $m$. The rank of $D(z, h)=h^{-1}[F(z+h)-F(z)]$ cannot exceed $2 m$, so that 
rank $F^{\prime}(z) \leqq 2 m$ by upper semicontinuity of rank. Hence, by (1) and (2),

$$
\begin{array}{r}
\left|h^{-1}[\operatorname{tr} F(z+h)-\operatorname{tr} F(z)]-\operatorname{tr} F^{\prime}(z)\right|=\left|\operatorname{tr}\left(D(z, h)-F^{\prime}(z)\right)\right| \\
\leqq \tau\left(D(z, h)-F^{\prime}(z)\right) \leqq 4 m \beta\left(D(z, h)-F^{\prime}(z)\right) .
\end{array}
$$

But the final term tends to zero as $h \rightarrow 0$, since $F(z)$ is analytic in norm.

4. TheOREM. If $F(z)$ is analytic and the rank of $F(z)$ is finite for all $z$ in $\Omega$, then $\Delta(z)=\operatorname{det}(I+F(z))$ is analytic.

Proof. Let $z_{0}$ be in $\Omega$, and set $F_{1}(z)=F(z)-F\left(z_{0}\right)$. For $z$ near $z_{0}$, $\beta\left(F_{1}(z)\right)<1 / 2$, so that $I+F_{1}(z)$ is invertible and

$$
I+F(z)=\left[I+F\left(z_{0}\right)\left(I+F_{1}(z)\right)^{-1}\right]\left[I+F_{1}(z)\right] .
$$

The determinant of the first factor is analytic, since the range of $F\left(z_{0}\right)\left(I+F_{1}(z)\right)^{-1}$ is contained in a fixed space [1, pp. 160-162] while $[1,(5.60)$, p. 46$]$

$$
\operatorname{det}\left(I+F_{1}(z)\right)=\exp \left\{\operatorname{tr} \log \left(I+F_{1}(z)\right)\right\}
$$

where

$$
\log \left(I+F_{1}(z)\right)=\sum_{k=1}^{\infty} \frac{(-1)^{k-1}}{k}\left(F_{1}(z)\right)^{k} .
$$

The right side of (6) converges uniformly in operator norm and contains a factor $F_{1}(z)$. Theorem 3 therefore applies to (6), and it follows that (5) is analytic.

5. CoROllaRY. If $F(z)$ is meromorphic and the rank of $F(z)$ is finite for all $z$ in $\Omega$, then $\Delta(z)=\operatorname{det}(I+F(z))$ and $\operatorname{tr} F(z)$ are meromorphic.

Proof. Let $z_{0}$ be a pole of $F(z)$ of order $p$. Then $\left(z-z_{0}\right)^{p} F(z)$ is analytic, and hence $\operatorname{tr} F(z)=\left(z-z_{0}\right)^{-p} \operatorname{tr}\left(z-z_{0}\right)^{p} F(z)$ is meromorphic, with the order of its pole not exceeding $p$. If we write $F(z)=P(z)+K(z)$ where $K\left(z_{0}\right)=0$ and $P(z)$ is a polynomial in $\left(z-z_{0}\right)^{-1}$, then we have near $z_{0}$

$$
\Delta(z)=\operatorname{det}\left(I+P(z)[I+K(z)]^{-1}\right) \operatorname{det}(I+K(z)) .
$$

The first factor is meromorphic since $P(z)$, being a polynomial, has its range contained in a fixed space; the second factor is analytic by Theorem 4.

6. TheOREM. In Theorem 4,

$$
\Delta^{\prime}(z) / \Delta(z)=\operatorname{tr}[I+F(z)]^{-1} F^{\prime}(z)
$$

provided that $\Delta(z)$ does not vanish identically. 
Proof. We first note that (7) applies to the first factor on the right side of (4), since the range of $F\left(z_{0}\right)\left(I+F_{1}(z)\right)^{-1}$ is contained in a fixed finite dimensional space [1, pp. 248-249]. It also applies to the second factor; for since

$$
\tau_{1}\left(F_{1}(z)^{k}\right) \leqq \tau\left(F_{1}(z)\right) \beta\left(F_{1}(z)\right)^{k-1} \leqq 2 m \beta\left(F_{1}(z)\right)^{k} \leqq\left(m / 2^{k-1}\right)
$$

the series (6) converges uniformly in trace norm, and we have

$$
\log \operatorname{det}\left(I+F_{1}(z)\right)=\operatorname{tr} \log \left(I+F_{1}(z)\right)
$$

$$
=\sum_{k=1}^{\infty} \frac{(-1)^{k-1}}{k} \operatorname{tr} F_{1}(z)^{k}
$$

where the series is uniformly convergent. Taking the trace of

$$
d F_{1}(z)^{k} / d z=F_{1}^{\prime} F_{1}^{k-1}+F_{1} F_{1}^{\prime} F^{k-2}+\cdots+F_{1}^{k-1} F_{1}^{\prime}
$$

(where $F_{1}$ and $F_{1}^{\prime}$ denote $F_{1}(z)$ and $F_{1}^{\prime}(z)$ respectively) and using Theorem 3, we find that

$$
d \operatorname{tr} F_{1}^{k}(z) / d z=k \operatorname{tr}\left(F_{1}(z)^{k-1} F_{1}^{\prime}(z)\right) .
$$

Differentiating (8) term by term, we obtain for the logarithmic derivative of $\operatorname{det}\left(I+F_{1}(z)\right)$

$$
\sum_{k=1}^{\infty}(-1)^{k-1} \operatorname{tr}\left(F_{1}(z)^{k-1} F_{1}^{\prime}(z)\right)=\operatorname{tr}\left\{\left(I+F_{1}(z)\right)^{-1} F_{1}^{\prime}(z)\right\} .
$$

If we now take determinants in (4) and apply these results, we obtain

$$
\begin{aligned}
\Delta^{\prime} / \Delta= & \operatorname{tr}\left\{F_{1}^{\prime}\left(I+F_{1}\right)^{-1}\right\} \\
& -\operatorname{tr}\left\{\left[I+F_{0}\left(I+F_{1}\right)^{-1}\right]^{-1} F_{0}\left(I+F_{1}\right)^{-1} F_{1}^{\prime}\left(I+F_{1}\right)^{-1}\right\} \\
= & \operatorname{tr}\left\{\left[I+F_{0}\left(I+F_{1}\right)^{-1}\right]^{-1} F_{1}^{\prime}\left(I+F_{1}\right)^{-1}\right\} \\
= & \operatorname{tr}\left\{\left[I+F_{0}+F_{1}\right]^{-1} F_{1}^{\prime}\right\} \\
= & \operatorname{tr}\left\{[I+F]^{-1} F^{\prime}\right\} .
\end{aligned}
$$

where $F_{0}=F\left(z_{0}\right)$.

\section{REFERENCES}

1. T. Kato, Perturbation theory for linear operators, Die Grundlehren der math. Wissenschaften, Band 132, Springer-Verlag, New York, 1966. MR 34 \#3324.

2. A. F. Ruston, On the Fredholm theory of integral equations for operators belonging to the trace class of a general Banach space, Proc. London Math. Soc. (2) 53 (1951), 109-124. MR 13, 138.

3. - A - A uerbach's theorem and tensor products of Banach spaces, Proc. Cambridge Philos. Soc. 58 (1962), 476-480. MR 29 \#2630.

University of Virginia, Charlottesville, Virginia 22901 